\title{
Oxalis Sect. Palmatifoliae (Oxalidaceae): Morfología de los GRANOS DE POLEN Y DIVERSIDAD DE LAS ORBÍCULAS
}

\author{
ALICIA LÓPEZ ${ }^{1 *}$ y SONIA ROSENFELDT²
}

\begin{abstract}
Resumen: La morfología del polen y las características de las orbículas fueron estudiadas en todas las especies de Oxalis sección Palmatifoliae, endémica del sur de Sudamérica, empleando microscopio óptico (M.O.) y microscopio electrónico de barrido (M.E.B.). Los granos de polen son 3-colpados, de forma prolato esferoidales, oblato esferoidales o esferoidales. La exina es microrreticulada. Lúmen circular a poligonal; disminuyendo de tamaño hacia los polos. Las orbículas están dispersas irregularmente en la superficie interna de las anteras; son generalmente aplanadas, redondeadas o con forma de "dona", de superficie lisa. La homogeneidad observada en la morfología de granos de polen de las especies de Oxalis sección Palmatifoliae refuerza la hipótesis de su origen monofilético y la variedad de tipos de orbículas podría contribuir a la diferenciación interespecífica.
\end{abstract}

Palabras clave: Argentina, Chile, Patagonia, Micromorfología, Oxalis.

Summary: Oxalis sect. Palmatifoliae (Oxalidaceae): pollen grains morphology and orbicules diversity. Pollen morphology and orbicules characteristics were studied by means of transmitted light microscopy (LM) and scanning electron microscopy (SEM) within five species of Oxalis section Palmatifoliae endemic from southern South America. Oxalis pollen grain is generally 3-colpate and the shape is prolate spheroidal, oblate spheroidal or spheroidal. The exine is microreticulate. The brochi are circular to polygonal; brochi decrease in size near the colpi. The orbicules are randomly dispersed on the inner surface of the anther locule. They are mostly plate-like, rounded shape, or doughnut, with smooth surface. The homogeneity observed in the pollen grains of all species of Oxalis section Palmatifoliae may reinforce the hypothesis of the monophyletic origin of the group, and the variability in orbicles types may contribute to the interspecific differentiation.

Key words: Argentina, Chile, Patagonia, Micromorphology, Oxalis.

\section{INTRODUCCIÓN}

Oxalis L. (Oxalidaceae) es un género cosmopolita que incluye ca. 500 especies. Está dividido en cuatro subgéneros, Oxalis L., Monoxalis (Small) Lourteig (1980), Trifidus Lourteig (1995) y Thamnoxys (Endl.) Reich. emend. Lourteig (2000) (Lourteig, 1994, 1995, 2000). El subgénero Oxalis

\footnotetext{
${ }^{1}$ Instituto de Botánica Darwinion (CONICET - ANCEFN), Labardén 200, CC 22, B1642HYD, San Isidro, Buenos Aires, Argentina. alopez@darwin.edu.ar. +54 11 47434800 int. 117

${ }^{2}$ Departamento de Biodiversidad y Biología Experimental. Facultad de Ciencias Exactas y Naturales. Universidad de Buenos Aires. Ciudad Universitaria. Pab. II. Buenos Aires, Argentina. C1428EHA. soniar@bg.fcen.uba.ar *Autor para correspondencia: alopez@darwin.edu.ar
}

incluye 17 secciones (Lourteig, 2000), la sección Palmatifoliae DC presenta los siguientes caracteres diagnósticos: acaulescente, hojas palmadas, pecioladas, peltatinervadas, multifolioladas (5 a 13 folíolos), aglandulares, flores dispuestas en cimas unifloras (raro bifloras). La sección incluye cinco especies: $O$. adenophylla Gillies ex Hook. \& Arn., O. enneaphylla Cav. y O. loricata Dusén endémicas de Argentina y Chile, y $O$. laciniata Cav. y O. morronei López \& Múlgura endémicas de Argentina (López et al., 2013).

Estudios palinológicos previos realizados en Oxalis incluyeron aspectos tales como morfología del grano de polen (Perveen \& Qaiser, 2003; Rosenfeldt \& Galati, 2007; Wan \& Chen, 2001), tipificación (Ghosh \& Verma, 1985), ontogenia (Herr, 1972; Guth \& Weller, 1986; Rosenfeldt 
\& Galati, 2005), formación de polen aberrante (Cerceau, 1995; Erdtman, 1952; Huynh, 1969 a, b; Rosenfeldt \& Galati, 2012), categorías de polen aberrante (Dreyer \& Van Wyk, 1998). Las orbículas también fueron empleadas en taxonomía de Oxalis (Rosenfeldt \& Galati, 2005, 2007, 2008).

En el contexto de un estudio integral de Oxalis sección Palmatifoliae que incluye revisión taxonómica (López et al., 2013), estudios citogenéticos (López \& Vaio, en prep.), y anatómicos, se presenta este trabajo que tiene como objetivos (1) proveer de una descripción de la morfología del polen de la sección Palmatifoliae, y (2) caracterizar la diversidad de orbículas presentes en este grupo endémico.

\section{Materiales y Métodos}

Las anteras fueron extraídas de ejemplares de herbario depositados en BAB y SI (Apéndice). Para microscopía óptica, se montaron los granos de polen en glicerol y los preparados se sellaron con parafina. Para microscopía electrónica de barrido, las anteras fueron tratadas con alcohol 100\% y posteriormente se dejaron secar. El metalizado fue realizado con oro-paladio por 3 minutos. Las fotomicrografías fueron obtenidas empleando el microscopio Zeiss Supra 40 FESEM. Para las descripciones se siguió la terminología general de Punt et al. (1994).

\section{Resultados y Discusión}

Oxalis sección Palmatifoliae (Fig. 1).

Granos de polen. Polen 3-colpado, prolato esferoidal, oblato esferoidal o esferoidal; ámbito redondeado o triangular. Exina microrreticulada, semitectada. Lumen redondeado a poligonal, con márgenes irregulares, el tamaño del retículo decrece hacia los colpos. Ornamentaciones del lumen a veces presente, variando en tamaño y densidad.

Oxalis adenophylla (Fig. 1 A-B): Exina homo reticulada. Lumen de contorno poligonal a circular con ornamentaciones aproximadamente esferoidales en alta densidad, conspicuas, parcialmente soldadas entre sí.

Oxalis enneaphylla (Fig. 1 D-E): Exina hetero reticulada. Lumen poligonal a irregular con ornamentaciones aproximadamente esferoidales, conspicuas, abundantes, algunas soldadas al muro.

Oxalis laciniata (Fig. $1 \mathrm{G}-\mathrm{H}$ ): Exina hetero reticulada. Lumen poligonal a circular con ornamento ausente o escaso.

Oxalis loricata (Fig. $1 \mathrm{~J}-\mathrm{K}$ ): Exina hetero reticulada. Lumen poligonal a irregular con escasas ornamentaciones de forma aproximadamente esferoidal, de inconspicuas.

Oxalis morronei (Fig. $1 \mathrm{M}-\mathrm{N}$ ): Exina homo reticulada. Lumen de contorno circular, con ornamentaciones ausentes o escasas.

Orbículas. Las orbículas están dispersas al azar en la superficie interna de las anteras. Tienen forma de placas, con contornos redondeados a irregulares, y superficies lisas (Fig. 1 C, F, I, L, O). Las variaciones en el tamaño y la forma pueden estar vinculadas al cambio en el ciclo hiperactivo de las células del tapete durante su desarrollo. Las orbículas pueden unirse entre sí resultando en agregados alargados con margen ondulado. Perforaciones superficiales están presentes en todas las especies examinadas, en $O$. loricata (Fig. $1 \mathrm{~L}$ ) las perforaciones atraviesan las orbículas dándole el aspecto de "dona". Siguiendo la clasificación de Rosenfeldt \& Galati (2008), la especie $O$. loricata presenta Orbículas Tipo II, las especies $O$. adenophylla y $O$. enneaphylla presentan un tipo intermedio entre el III y IV (en donde se observa algunos agregados de orbículas en forma alargada pero no tan numerosos como para definirlas en el tipo III), y las especies $O$. laciniata y $O$. morronei presentan orbículas del Tipo IV.

De acuerdo al presente estudio, las especies de Oxalis sección Palmatifoliae tienen polen 3-colpado, prolato esferoidal, oblato esferoidal o esferoidal, lo que coincide con la mayoría de las especies de Oxalis descriptas (Rosenfeldt \& Galati, 2007).

La monofilia de la sección Palmatifoliae fue propuesta con anterioridad en base a estudios moleculares (Heibl \& Renner, 2012) y morfológicos (López et al., 2013), la homogeneidad observada en los granos de polen podría reforzar la hipótesis del origen monofilético del grupo y la variedad de tipos de orbículas podría contribuir a la diferenciación interespecífica. 

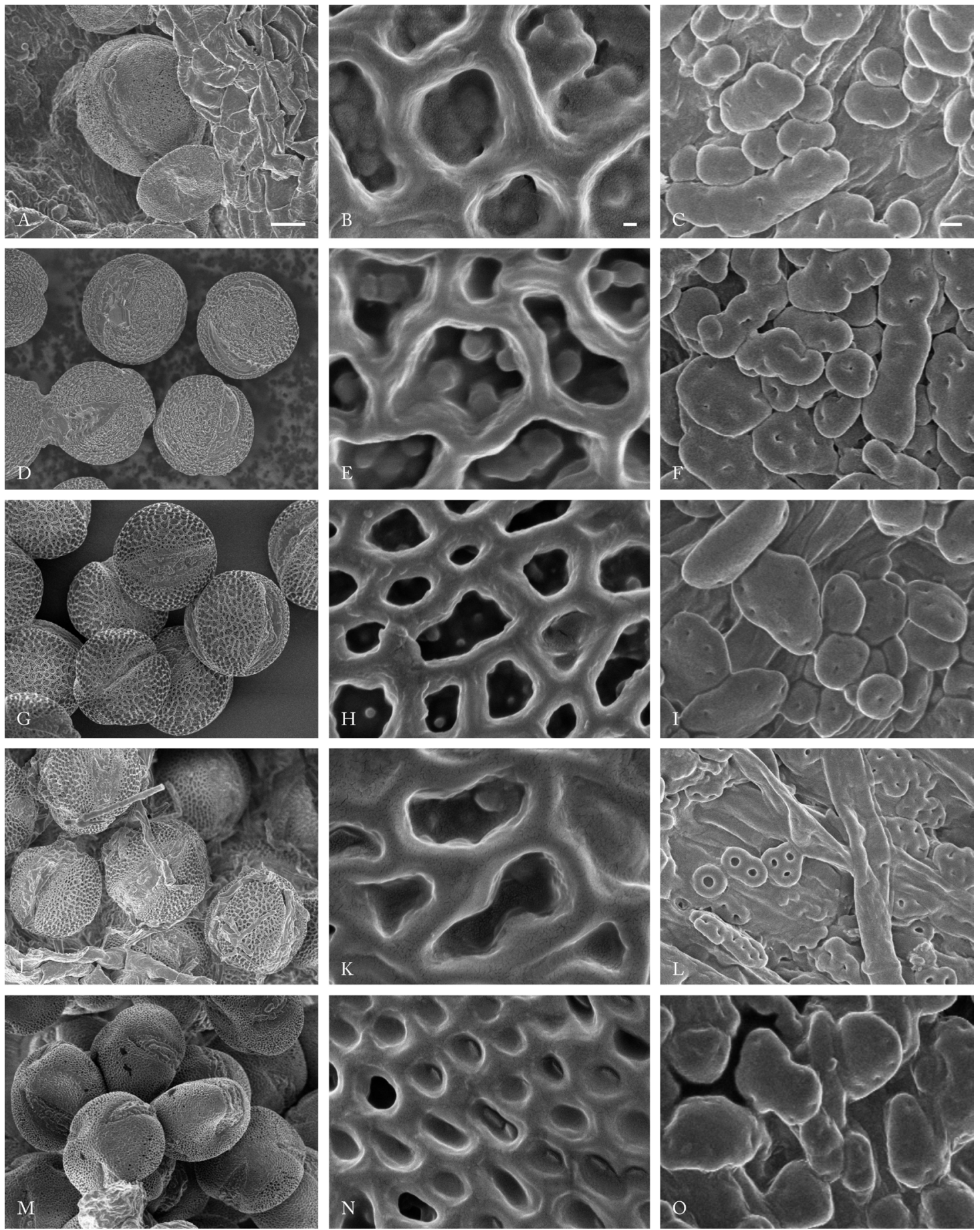

Fig. 1. Microfotografías al MEB de los granos de polen y las orbículas en especies de Oxalis sección Palmatifoliae. (A-B-C) Oxalis adenophylla, (D-E-F) Oxalis enneaphylla, (G-H-I) Oxalis laciniata, (J-K-L) Oxalis loricata, (M-N-O) Oxalis morronei. (A-D-G-J-M). Vistas generales de los granos de polen, escala $=10 \mu \mathrm{m}(\mathrm{B}-\mathrm{E}-\mathrm{H}-\mathrm{K}-\mathrm{N})$. Detalle de la exina, escala=200 nm. (C-F-I-L-O). Vista general de las orbículas, escala $=1 \mu \mathrm{m}$. 


\section{Agradecimientos}

A los curadores de BAB y SI por los préstamos y autorización para usar material de herbario; a la ANPCyT, Préstamo BID-PICT-2013-0291; a los revisores por sus comentarios que mejoraron el manuscrito.

\section{ApÉndice}

Especímenes examinados. Oxalis adenophylla Gillies ex Hook \& Arn: ARGENTINA. Mendoza: Dept. Malargüe, Paso Pehuenche, límite con Chile, 24 November 2010, F.O. Zuloaga et al. 12442 (SI); Santa Cruz: Dept. Lago Buenos Aires, camino fronterizo, al N del Paso R. Roballos, rumbo a los Antiguos, 24 January 2003, M.J. Belgrano 85 (SI); Oxalis enneaphylla Cav. ARGENTINA. Santa Cruz: Ea. Guaken-Aike, camino al puesto La Carlina, 9 November 1977, TBPA 2399 (SI); Dept. Lago Argentino, El Calafate, Co. Huiliche excursión 4 x 4, laberinto 214 December 2001, C. Guerrido 425 (SI). Tierra del Fuego, Ea. La Sara, a 60 km de Río Grande 8 December 1966, Kreinohn 535 (SI); Oxalis laciniata Cav. ARGENTINA. Santa Cruz: Dept. Lago Buenos Aires, Perito Moreno, November 1973, C. Suarez 813 (SI); Dept. Lago Argentino, El Calafate, Campo Calafate, camino a CEJL, 13 November 1999, C. Guerrido et al. 136 (SI); Co. Huyliche Excursión 4x4 Laberinto 1, 11 January 2001, C. Guerrido et al. 355 (SI); Dept. Magallanes, San Julián, 5 November 1955, J. Blake 447 (SI); Oxalis loricata Dusén. CHILE. Prov. Tierra del Fuego, $15 \mathrm{~km} \mathrm{~N}$ of Porvenir, Lag. de los Cygnes, 6 November 1971, D.M. Moore 2333 (SI), Porvenir, Co. Pirámide, 28 January 1939, I. von Rentzell 6107 (SI); Oxalis morronei López and Múlgura ARGENTINA: Santa Cruz: Dept. Lago Buenos Aires, meseta del lago, $35 \mathrm{~km}$ NW del casco ea. La Vizcaína, 10 December 1986, M.I. Sánchez et al. 567 (BAB); RP 41, camino de Los Antiguos a Paso Río Roballos, 9 January 2011. L.M. Zavala Gallo et al. 204 (SI).

\section{Biblografía}

CERCEAU, M-T. 1995. Palynologie. Bradea 6: 392-395. DE CANDOLlE, A. P. 1824. Oxalideae. In A.P. DE
CANDOLLE (ed.), Prodromus Systematis Naturalis Regni Vegetabilis 1, 689-702. Treutell and Würst. Paris.http://dx.doi.org/10.5962/bhl.title.286

DREYER, L. L. \& A. E. VAN WYK. 1998. Aberrant pollen in Southern Africa Oxalis (Oxalidaceae). Grana 37: 337-342.

ERDTMAN, G. 1952. Pollen Morphology and Plant Taxonomy. Angiosperms (An Introduction to Palynology I): Waltham, Mass., U.S.A. The Chronica Botanica Co.

GHOSH, T. K. \& D. K.VERMA. 1985. Pollen types in Oxalis. Acta Bot Indica 13: 148-151.

GUTH, C.J. \& S. G. WELLER. 1986. Pollination, fertilization and ovule abortion in Oxalis magnifica. Am J Bot 73: 246-257.

HEIBL, C. \& S. RENNER. 2012. Distribution Models and a Dated Phylogeny for Chilean Oxalis Species Reveal Occupation of New Habitats by Different Lineages, not Rapid Adaptive Radiation. Systematic Biology 61: 823-834.

HERR, J.M. JR. 1972. An extended investigation on the megagametophyte in Oxalis corniculata L. In: MURTY, Y. S., JOHORI, B. M., MOHAN RAM, H. Y. \& T. M. VARGHESE (eds.), Advances in plant morphology. Sarita Prkasham. Meerut (India), pp 92-101.

HUYNH, K.L. 1969a. Etude du pollen des Oxalidaceae. II. (suite et fin) Palynotaxonomie des Oxalis sudafricains- Considerations générales. Bot $\mathrm{Jb}$. 89: 305-334.

HUYNH, K.L. 1969b. Etude du pollen des Oxalidaceae. I. Morphologie generale - palyno- taxonomie des Oxalis Americains. Bot Jb. 89: 272-303.

LÓPEZ, A., PANSERI, A. F. \& E. URTUBEY. 2013. Revision of genus Oxalis L. section Palmatifoliae (Oxalidaceae). Phytotaxa 138: 1-14.

LOURTEIG, A. 1994. Oxalis L. subgénero Thamnoxys (Endl.) Reiche emend. Lourt. Bradea 7: 1-199.

LOURTEIG, A. 1995. Oxalis L. Subgenus Trifidus Lourt. n. subgen. Bradea 6: 389-395.

LOURTEIG, A. 2000. Oxalis L. subgéneros Monoxalis (Small) Lourt., Oxalis y Trifidus Lourt. Bradea 7: 201-629.

PERVEEN, A. \& M. QAISER. 2003. Pollen Flora of Pakistan XXII. Oxalidaceae. Pakistan J Bot 35: 3-6.

PUNT, W., BLACKMORE, S., NILSSON, S. \& A. LE THOMAS. 1994. Glossary of Pollen and Spore Terminology. LPP Contributions series $\mathrm{N}^{\circ}$ 1.LPP Foundation. Utrech.

Recibido el 09 de febrero de 2015, aceptado el 28 de abril de 2015. 